\title{
Comparison between Hydroponic and Soil-Grown Strawberries: Sensory Attributes and Correlations with Nutrient Content
}

\author{
Chenin Treftz, Fannie Zhang, Stanley T. Omaye \\ Environmental Sciences and Health Graduate Program, Agriculture, Nutrition and Veterinary Sciences \\ Department, University of Nevada, Reno, USA \\ Email: chenin.treftz@gmail.com,omaye@unr.edu
}

Received 25 September 2015; accepted 16 November 2015; published 19 November 2015

Copyright @ 2015 by authors and Scientific Research Publishing Inc.

This work is licensed under the Creative Commons Attribution International License (CC BY). http://creativecommons.org/licenses/by/4.0/

c) (i) Open Access

\begin{abstract}
Hydroponic growing methods are growing in popularity and seem to have numerous benefits (i.e., environmental, increased product yields, year round growing) compared to soil grown crops. Although these advantages are attractive, they do not guarantee a high quality product. Taste is a driver of consumer acceptance; therefore, sensory analysis of the hydroponic product will be an important indicator in its success. In this study, we evaluated the sensory differences and preferences in hydroponically grown and soil-grown strawberries (Fragaria $x$ ananassa) using unspecified discriminatory and preference analyses, and descriptive testing correlated with nutrition content data. Most (87\%) of participants could identify differences between hydroponically and soil grown strawberries and $70 \%$ preferred the hydroponically grown strawberry $(p=0.06)$. The nutrient composition of the strawberries significantly influenced several sensory analysis categories (sweetness, overall flavor and overall taste $(p<0.05)$ ). The use of sensory studies in relation to consumer acceptance and nutrient quality will be an important factor to consider for exploring growing methods and techniques in hydroponic technology.
\end{abstract}

\section{Keywords}

Hydroponic, Sensory Evaluation, Sustainable Foods, Strawberry, Greenhouse

\section{Introduction}

Consumers are becoming more aware of nutritious and sustainable food options. Soilless grown fruits and vegetables have been gaining momentum and have caught the attention of many producers, consumers and scientists

How to cite this paper: Treftz, C., Zhang, F. and Omaye, S.T. (2015) Comparison between Hydroponic and Soil-Grown Strawberries: Sensory Attributes and Correlations with Nutrient Content. Food and Nutrition Sciences, 6, 1371-1380. 
because of the plethora of benefits offered by soilless growing methods [1]. Hydroponics is one type of soilless growing method, among others, are aquaponics, aeroponics and fogponics. For soilless products to be successful, the environmental, nutritional quality and sensory attributes must be equal to or better than soil grown produce. From an environmental perspective, soilless food production offers increased yields, higher plant survival rates, decreased water use and decreased pesticide use. From a producer standpoint, these environmental benefits offer greater profits resulting from increased yields and decreased use of resources (i.e., water, pesticides, labor). Nutritionally, some studies have indicated that soilless growing methods have superior nutritional quality, while others indicate no significant differences [2]-[4].

Few studies have investigated the sensory comparison between hydroponically grown produce and soil grown produce. Sensory evaluation can be affected by several factors such as genetic composition, pre-harvest factors (light, temperature, moisture and wind) and post-harvest factors [5]-[7]. Other influences include price, branding and the mood of the consumer has been shown to affect the sensory evaluation of a product [8]-[10]. All of these are important factors to consider when evaluating a hydroponically grown product for sensory attributes. Previous sensory analyses of hydroponically grown produce have predominately focused on lettuce or tomatoes. Some of these studies indicate higher ratings for hydroponically grown produce, while others indicate no significant differences. Little research has focused on sensory evaluation of hydroponically grown strawberries. Strawberries are rich in health promoting bioactive compounds and the consumption of them is associated with decreased risk for obesity, cardiovascular disease and certain types of cancer [11] [12].

The goal of this study was to evaluate the sensory differences in hydroponically grown and soil grown strawberries (Fragaria x ananassa). First, unspecified discriminatory and preference tests were conducted. Then, descriptive evaluation of 13 sensory attributes between the hydroponic and soil grown strawberries were conducted, and determined if an association existed between nutritional composition of the strawberries.

\section{Methods}

\subsection{Growing Conditions}

The growing conditions have been described previously [3]. Briefly, soil and hydroponically grown strawberries were grown at the University of Nevada, Reno (UNR) Experimental Station. During the growing season, the greenhouse temperature was kept at $70^{\circ} \mathrm{F}$ during the day and $60^{\circ} \mathrm{F}$ at night. The humidity averaged at $30 \%$. Hydroponic and soil strawberries were randomized within the available space in the greenhouse. Soil grown strawberries were planted in a 1:1 ratio of Miracle-Gro ${ }^{\circledR}$ potting soil (Maryville, $\mathrm{OH}$ ) and Nevada top soil. The strawberries were watered by a drip irrigation system three times weekly. General Hydroponics Flora Series (Sebastopol, CA) was the nutrient solution used for the hydroponic strawberries and nutrient ratios were adjusted according to the manufacturer instructions during different stages of plant development. The $\mathrm{pH}$ and parts per million concentration (ppm) of the nutrient for the strawberries was maintained between 6.0 - 6.4 and 400 ppm, respectively. These strawberries were adjusted three times weekly, as needed.

\subsection{Harvesting and Sample Preparation}

Strawberries were harvested between 7 AM and 8 AM by hand when they reached $100 \%$ visual red surface color. The strawberries were placed in a plastic laboratory bag and immediately transported to the UNR sensory laboratory. UNR sensory evaluation booths were designed according to the American Society of Testing Materials standards. The sensory evaluation laboratory booths were 34 " $\times 60$ " $\times 72$ " and were designed to ensure participation privacy during testing. These booths were built with an 11 ” sliding door serving hatch to serve participants.

The strawberries used for sensory evaluation were harvested on the same day they were evaluated by participants. These strawberries were rinsed with tap water before serving and allowed to dry on a paper towel. Berries were served at room temperature in two ounce, clear plastic cups coded with a three digit random number. The average sample size for each portion was bite size, approximately 10 grams.

\subsection{Participant Selection}

The study was approved by the UNR Institutional Review Board (project number: 583149-1). Participants were untrained in sensory evaluation and were undergraduate or graduate students at UNR. Participants were re- 
cruited through word of mouth as well as undergraduate nutrition classes and asked to come to the UNR sensory analysis laboratory to give their preferences on locally grown strawberries.

\subsection{Surveys for Sensory Evaluation}

Unspecified discriminatory tests, unspecified preference tests, and descriptive tests using 13 descriptive attributes were used to evaluate the strawberries. The discriminatory test used to determine a difference in the strawberries was the tetrad test. The students were presented with four samples (two hydroponically grown strawberries and two soil grown strawberries) and were asked to group the samples into two groups of two based on similarity. The tetrad test offers advantages compared to other discriminatory tests because it allows for increased statistical power explained by the Thurstonian theory [13]. The tetrad also has advantages because it decreases effect size and reduces unexplained variations within the samples, therefore using less panelists and samples [13].

Preference tests were conducted after the discriminatory test was completed. Volunteers participating in the preference tests were given two coded samples (one hydroponically grown berry and one soil grown berry). The participants were asked to circle the sample they preferred, or to circle "no preference" if they did not have a preference between the two samples.

Descriptive analysis was conducted using a 5-point hedonic scale using a "smiley face" which depicted cartoon faces with smiles to frowns [14]. The cartoon faces were labeled from "very satisfied" to "vey unsatisfied". The following attributes were evaluated: overall color, color uniformity, overall appearance, overall aroma, aroma intensity, amount of sweetness, amount of sourness, fruit juiciness, overall taste, fruit firmness, overall texture, overall mouthfeel and overall flavor.

\section{Statistical Analysis}

Data for the tetrad and preference tests were analyzed using IBM SPSS Statistics ${ }^{\circledR}$, version 23.0 (IBM, Armonk, New York, USA). Data for the correlation analysis was analyzed using SAS ${ }^{\circledR}$, version 9.4 (Cry, N.C., USA). The tetrad test was analyzed by calculating the test statistic (d') and used to determine the effect size [15]. A d' of 1 can be considered as a threshold value for psychophysics [16]. Sample sizes for the tetrad test was calculated at $\alpha$-level $=0.05$ and power $=0.8$ [17]. Preference tests were first evaluated by chi-square analysis and then by binominal statistics. Descriptive tests were first compared using the independent t-test $(\mathrm{p}<0.05)$ and correlated to existing nutritional data ordered linear regression. Results were adjusted for sex, age, ethnicity, and fresh strawberry consumption.

\section{Results}

\subsection{Discrimination Test}

Sensory evaluation for the discrimination determined if volunteers could detect an overall difference between hydroponically and soil grown strawberries. Descriptive characteristics for all tests are outlined in Table 1 . The panel included 15 participants, aged 17 - 64 with the majority of volunteers between the ages 17 - 29. Results indicated, out of 15 participants, 87\% completed the questionnaire correctly. By using the Thurstonian theory, a d' was calculated at 2.0, and with a threshold of d' $=1.0$, the majority of participants were generally able to discriminate between hydroponically and soil grown strawberries.

\subsection{Preference Test}

Preference test data results are outlined in Table 2. The chi-square was first used to determine significance in distributions between prefer hydroponic, prefer soil and no preference $(\mathrm{p}<0.05)$. Zero participants chose the "no preference" option and binomial statistics were used to determine significance between preference for hydroponically grown strawberries and soil grown strawberries. For the strawberries, $70 \%$ of participants indicated that they preferred hydroponically grown strawberries compared to the soil grown strawberries; however, the preference was not significantly different $(\mathrm{p}=0.06)$.

\subsection{Descriptive Test}

The results for the descriptive tests between hydroponically and soil grown strawberries are outlined in Table 3 . 
Table 1. Demographics of participants participating in the discrimination test, preference test and descriptive test.

\begin{tabular}{cc}
\hline \multicolumn{2}{c}{ Tetrad Test $(\mathrm{N}=15)$} \\
\cline { 2 - 3 } Number & Percent \\
\hline
\end{tabular}

Sex

$\begin{array}{lll}\text { Male } & 7 & 47 \\ \text { Female } & 8 & 53\end{array}$

Age

$\begin{array}{lll}17-29 & 7 & 47 \\ 30-49 & 7 & 46 \\ 50-64 & 1 & 7 \\ 65+ & 0 & 0\end{array}$

Fresh Berry Consumption

More than once per day 0

Once a day 0

Two to four times per week $\quad 3 \quad 20$

At least once per week $\quad 5$

$\begin{array}{lll}\text { Once per month } & 7 & 47\end{array}$

Rarely $\quad 0$

Ethnicity

$\begin{array}{lcc}\text { American Indian } & 0 & 0 \\ \text { Asian } & 4 & 27 \\ \text { African American } & 0 & 0 \\ \text { Hispanic } & 3 & 20 \\ \text { White } & 8 & 53 \\ \text { Other } & 0 & 0 \\ & \text { Preference Test }(\mathrm{N}=20) \\ & \text { Number } & \text { Percent }\end{array}$

Sex

$\begin{array}{lcc}\text { Male } & 8 & 40 \\ \text { Female } & 12 & 60\end{array}$

Age

$\begin{array}{lcc}17-29 & 11 & 55 \\ 30-49 & 6 & 30 \\ 50-64 & 2 & 10 \\ 65+ & 1 & 5\end{array}$

Fresh Berry Consumption

More than once per day 


\section{Continued}

$\begin{array}{lll}\text { Once a day } & 0 & 0 \\ \text { Two to four times per week } & 3 & 15 \\ \text { At least once per week } & 7 & 35 \\ \text { Once per month } & 5 & 25 \\ \text { Rarely } & 4 & 20\end{array}$

Ethnicity

\begin{tabular}{lcc} 
American Indian & 1 & 5 \\
Asian & 4 & 20 \\
African American & 0 & 0 \\
Hispanic & 2 & 10 \\
White & 13 & 65 \\
Other & 0 & 0 \\
\hline & Descriptive Survey $(\mathrm{N}=20)$ \\
\hline & Number & Percent
\end{tabular}

Sex

$\begin{array}{lcc}\text { Male } & 9 & 45 \\ \text { Female } & 11 & 55\end{array}$

Age

$17-29$

15

75

$30-49$

3

15

$50-64$

1

$65+$

Fresh Berry Consumption

More than once per day

Once a day

1

Two to four times per week

At least once per week

3

Once per month

Rarely

Ethnicity

\begin{tabular}{lcc} 
American Indian & 0 & 0 \\
Asian & 4 & 20 \\
African American & 1 & 5 \\
Hispanic & 6 & 30 \\
White & 9 & 45 \\
Other & 0 & 0 \\
\hline
\end{tabular}


Table 2. Preference test results between hydroponically grown berries and soil grown berries.

\begin{tabular}{cccccc}
\hline & Number & $\begin{array}{c}\text { Preferred Hydroponically } \\
\text { Grown }\end{array}$ & $\begin{array}{c}\text { Preferred Soil } \\
\text { Grown }\end{array}$ & $\begin{array}{c}\text { Proportion preferred } \\
\text { hydroponic }\end{array}$ & p \\
\hline Strawberries & 20 & 14 & 6 & $70 \%$ & 0.06 \\
\hline
\end{tabular}

Table 3. Sensory analysis results of hydroponically compared to soil grown strawberries.

\begin{tabular}{ccccc}
\hline & Hydroponically Grown & Soil Grown & $\mathrm{t}$ & $\mathrm{p}$ \\
\hline Overall Color & $3.7 \pm 0.2$ & $3.3 \pm 0.2$ & 1.4 & 0.163 \\
Color Uniformity & $3.8 \pm 0.2$ & $3.6 \pm 0.2$ & 1.2 & 0.235 \\
Overall Appearance & $3.5 \pm 0.2$ & $3.4 \pm 0.3$ & 0.3 & 0.758 \\
Overall Aroma & $4.6 \pm 0.1$ & $3.3 \pm 0.2$ & 4.8 & $<0.001$ \\
Aroma Intensity & $4.3 \pm 0.1$ & $3.1 \pm 0.2$ & 4.4 & $<0.001$ \\
Amount of Sweetness & $3.7 \pm 0.2$ & $3.8 \pm 0.2$ & 0.3 & 0.725 \\
Amount of Sourness & $3.7 \pm 0.1$ & $3.6 \pm 0.2$ & 0.6 & 0.547 \\
Fruit Juiciness & $4.4 \pm 0.2$ & $4.0 \pm 0.2$ & 1.6 & 0.100 \\
Overall Taste & $3.8 \pm 0.2$ & $3.7 \pm 0.2$ & 0.2 & 0.734 \\
Fruit Firmness & $3.9 \pm 0.2$ & $4.1 \pm 0.2$ & 0.6 & 0.517 \\
Overall Texture & $4.2 \pm 0.2$ & $4.0 \pm 0.2$ & 0.6 & 0.541 \\
Overall Mouth Feel & $3.9 \pm 0.2$ & $3.8 \pm 0.2$ & 0.6 & 0.580 \\
Overall Flavor & $4.0 \pm 0.2$ & $3.6 \pm 0.2$ & 1.1 & 0.194
\end{tabular}

Means \pm standard deviations are based 20 judges' scores on 5 -point hedonic scale $(5=$ very satisfied, $4=$ satisfied, $3=$ neutral, $2=$ unsatisfied, $1=$ very unsatisfied).

Of the 13 attributes studied, overall aroma and aroma intensity were the only attributes that reached statistical significance $(\mathrm{p}<0.05)$. Hydroponically grown strawberries showed higher mean ratings for these two categories. Among the other 11 attributes assessed, nine of the attributes showed higher mean scores for hydroponically grown strawberries compared to soil grown strawberries. Overall appearance and fruit firmness showed higher ratings for soil grown strawberries compared to hydroponically grown strawberries; however, results were not significant.

\subsection{Correlation between Sensory Analysis Data and Nutrient}

Methods and quantification of nutrient composition of the soil and hydroponically grown strawberries have been previously reported [3]. Additional nutrient data was collected for matching the correlational analysis. The updated results are reported in Table 4. It was assumed the nutrient data matched the sample for sensory analysis. For the soil grown strawberries, the correlational analysis indicated no significant association between sourness, appearance, aroma, color uniformity, fruit firmness, overall mouthfeel, fruit juiciness, or overall texture (Table 5). Significant associations were observed with amount of sweetness and ascorbic acid, $\alpha$-tocopherol, $\%$ moisture and Brix. Aroma intensity and overall color were significantly correlated with ascorbic acid. Overall flavor was significantly associated with ascorbic acid, $\alpha$-tocopherol and percent moisture. Overall taste was significantly associated with ascorbic acid and $\alpha$-tocopherol. For the hydroponically grown strawberries, the correlational analysis indicated no significant association between sourness, appearance, aroma, aroma intensity, color, color uniformity, fruit firmness, fruit juiciness, or overall texture (Table 6). Significant observations were observed with amount of sweetness and moisture content, overall mouthfeel and glucose. Overall taste and $\alpha$-tocopherol, percent moisture and fructose were also significantly associated. 
Table 4. Selected nutrients, Brix and \% moisture content of soil and hydroponic strawberries (mean \pm SD).

\begin{tabular}{|c|c|c|c|c|}
\hline & Hydroponic Strawberries & Soil Strawberries & $\mathrm{t}$ & $\mathrm{p}$ \\
\hline Brix (\%) & $7.6 \pm 0.29$ & $8.5 \pm 0.23$ & 1.39 & 0.17 \\
\hline \multirow[t]{2}{*}{$\%$ moisture } & $86.9 \pm 1.36$ & $88.6 \pm 0.61$ & 1.36 & 0.18 \\
\hline & Hydroponic Strawberries & Soil Strawberries & $\mathrm{t}$ & $\mathrm{p}$ \\
\hline Ascorbic Acid (mg/100g) & $32.32 \pm 1.27$ & $18.62 \pm 0.92$ & 8.82 & $<0.0001$ \\
\hline$\alpha$-tocopherol (mg/100g) & $1.80 \pm 0.12$ & $0.99 \pm 0.10$ & 5.11 & $<0.0001$ \\
\hline \multirow[t]{2}{*}{ Total phenolics (mg/100g) } & $344 \pm 6.72$ & $268 \pm 2.90$ & 18.76 & $<0.0001$ \\
\hline & Hydroponic Strawberries & Soil Strawberries & $\mathrm{t}$ & $\mathrm{p}$ \\
\hline Ructose (g/100g) & $2.89 \pm 0.06$ & $5.10 \pm 0.15$ & 14.27 & $<0.0001$ \\
\hline Glucose (g/100g) & $1.67 \pm 0.03$ & $4.43 \pm 0.06$ & 36.92 & $<0.0001$ \\
\hline
\end{tabular}

$\mathrm{N}=20$ with 3 replicates.

Table 5. Soil grown strawberries sensory ratings vs. nutritional attributes (odds ratio point estimates).

\begin{tabular}{|c|c|c|c|c|c|c|c|c|c|c|c|c|c|c|}
\hline Nutrient & Sourness & $\begin{array}{c}\operatorname{Pr}> \\
\text { Chi Sq }\end{array}$ & $\begin{array}{c}\text { Overall } \\
\text { appearance }\end{array}$ & \multicolumn{2}{|c|}{$\begin{array}{c}\operatorname{Pr}> \\
\text { Chi Sq }\end{array}$} & Sweetness & $\begin{array}{c}\operatorname{Pr}> \\
\text { Chi Sq }\end{array}$ & Aroma & $\begin{array}{c}\mathrm{Pr}> \\
\text { Chi Sq }\end{array}$ & \multicolumn{2}{|c|}{$\begin{array}{l}\text { Aroma } \\
\text { intensity }\end{array}$} & $\begin{array}{c}\operatorname{Pr}> \\
\text { Chi Sq }\end{array}$ & Color & $\begin{array}{c}\operatorname{Pr}> \\
\text { Chi Sq }\end{array}$ \\
\hline $\begin{array}{l}\text { Ascorbic } \\
\text { Acid }\end{array}$ & 0.857 & 0.2744 & 1.234 & \multicolumn{2}{|c|}{0.1356} & 595 & 0.022 & 0.801 & 0.1338 & \multicolumn{2}{|c|}{0.677} & 0.0254 & 1.996 & 0.0064 \\
\hline Tocopherol & 0.035 & $* *$ & 0.473 & \multicolumn{2}{|c|}{0.4978} & 03 & 0.0101 & 0.325 & 0.3268 & \multicolumn{2}{|c|}{0.129} & 0.0999 & 10.062 & 0.0704 \\
\hline Moisture & 0.871 & 0.5433 & 1.138 & \multicolumn{2}{|c|}{0.5502} & 2.293 & 0.015 & 0.886 & 0.5876 & \multicolumn{2}{|c|}{1.183} & 0.4586 & 1.006 & 0.9782 \\
\hline Glucose & 0.08 & 0.2906 & 1.225 & \multicolumn{2}{|c|}{0.9253} & 0.158 & 0.4468 & 12.544 & 0.2807 & \multicolumn{2}{|c|}{4.355} & 0.5131 & 1.891 & 0.7723 \\
\hline Fructose & 2.547 & 0.365 & 3.344 & \multicolumn{2}{|c|}{0.2211} & 0.253 & 0.1991 & 1.004 & 0.9966 & \multicolumn{2}{|c|}{0.431} & 0.3927 & 0.654 & 0.6488 \\
\hline Brix & 0.942 & 0.805 & 1.37 & \multicolumn{2}{|c|}{0.203} & 0.535 & 0.0408 & 0.694 & 0.1572 & \multicolumn{2}{|c|}{0.779} & 0.3024 & 2.166 & $* *$ \\
\hline Nutrient & $\begin{array}{c}\text { Color } \\
\text { Uniformity }\end{array}$ & $\begin{array}{c}\operatorname{Pr}> \\
\text { Chi Sq }\end{array}$ & $\begin{array}{l}\text { Overall } \\
\text { flavor }\end{array}$ & $\begin{array}{l}\mathrm{Pr}> \\
\text { Chi Sq }\end{array}$ & $\begin{array}{c}\text { Fruit } \\
\text { firmness }\end{array}$ & $\begin{array}{l}\mathrm{Pr}> \\
\text { Chi Sq }\end{array}$ & $\begin{array}{c}\text { Overall } \\
\text { Mouthfeel }\end{array}$ & $\begin{array}{c}\operatorname{Pr}> \\
\text { Chi Sq }\end{array}$ & $\begin{array}{c}\text { Fruit } \\
\text { Juiciness }\end{array}$ & $\begin{array}{c}\text { Pr }> \\
\text { Chi Sq }\end{array}$ & $\begin{array}{l}\text { Overall } \\
\text { Taste }\end{array}$ & $\begin{array}{c}\operatorname{Pr}> \\
\text { Chi Sq }\end{array}$ & Texture & $\begin{array}{c}\mathrm{Pr}> \\
\text { Chi Sq }\end{array}$ \\
\hline $\begin{array}{l}\text { Ascorbic } \\
\text { Acid }\end{array}$ & 44.35 & 0.3999 & 0.654 & 0.0476 & 0.95 & 0.7197 & 0.836 & 0.2193 & 1.037 & 0.8092 & 0.563 & 0.039 & 0.915 & 0.5411 \\
\hline Tocopherol & 93.955 & 0.0681 & 0.005 & 0.0107 & 0.427 & 0.4599 & 0.016 & $* *$ & 1.245 & 0.8608 & 0.004 & 0.0138 & 0.035 & $* *$ \\
\hline $\begin{array}{c}\text { Total } \\
\text { Phenolics }\end{array}$ & 0.923 & 0.2922 & 1.035 & 0.5305 & 0.954 & 0.3499 & 1.075 & 0.1867 & 0.995 & 0.9279 & 1.052 & 0.4484 & 1.061 & 0.2758 \\
\hline Moisture & 0.831 & 0.5106 & 3.006 & 0.0219 & 1.434 & 0.2269 & 1.286 & 0.294 & 0.506 & 0.0726 & 1.259 & 0.3928 & 1.238 & 0.3812 \\
\hline Glucose & 8.811 & 0.4019 & 5.898 & 0.4825 & 0.052 & 0.2169 & 0.314 & 0.6197 & 199.339 & 0.0894 & 2.031 & 0.7813 & 0.058 & 0.2606 \\
\hline Brix & 2.962 & 0.1754 & 1.315 & 0.2964 & 1.427 & 0.1746 & 1.093 & 0.7161 & 0.749 & 0.2918 & 1.128 & 1.853 & 1.107 & 0.6797 \\
\hline
\end{tabular}

${ }^{* *}$ Indicates $\mathrm{p}>0.05$, globe model is not significant. 
Table 6. Soil grown strawberries sensory ratings vs. nutritional attributes (odds ratio point estimates).

\begin{tabular}{|c|c|c|c|c|c|c|c|c|c|c|c|c|c|c|}
\hline Nutrient & Sourness & $\begin{array}{c}\mathrm{Pr}> \\
\text { Chi Sq }\end{array}$ & $\begin{array}{c}\text { Overall } \\
\text { appearance }\end{array}$ & & $\begin{array}{l}> \\
\mathrm{Sq}\end{array}$ & etness & $\begin{array}{c}\mathrm{Pr}> \\
\text { Chi Sq }\end{array}$ & Aroma & $\begin{array}{c}\mathrm{Pr}> \\
\text { Chi Sq }\end{array}$ & $\begin{array}{l}\text { Arom } \\
\text { intensi }\end{array}$ & \multicolumn{2}{|c|}{$\begin{array}{c}\operatorname{Pr}> \\
\text { Chi Sq }\end{array}$} & Color & $\begin{array}{c}\operatorname{Pr}> \\
\text { Chi Sq }\end{array}$ \\
\hline $\begin{array}{l}\text { Ascorbic } \\
\text { Acid }\end{array}$ & 1.06 & 0.616 & 0.87 & \multicolumn{2}{|c|}{0.1981} & 89 & 0.3242 & 1.08 & 0.6587 & 0.94 & \multicolumn{2}{|c|}{0.5455} & 0.90 & 0.293 \\
\hline Tocopherol & 8.80 & 0.1822 & 0.18 & \multicolumn{2}{|c|}{0.2521} & 09 & 0.125 & 0.90 & 0.9566 & 0.44 & \multicolumn{2}{|c|}{0.5824} & 0.14 & 0.2146 \\
\hline Moisture & 0.62 & 0.1756 & 1.82 & \multicolumn{2}{|c|}{0.1278} & 93 & 0.0219 & 3.68 & 0.6137 & 75.84 & \multicolumn{2}{|c|}{0.4572} & 1.21 & 0.4208 \\
\hline Glucose & 43.74 & 0.5573 & * & \multicolumn{2}{|c|}{0.3273} & 19 & 0.7595 & * & 0.6764 & 12.82 & \multicolumn{2}{|c|}{0.6889} & $*$ & 0.2318 \\
\hline Fructose & 0.45 & 0.8136 & $*$ & \multicolumn{2}{|c|}{0.069} & 5.29 & 0.2195 & * & 0.539 & 4.81 & \multicolumn{2}{|c|}{0.6459} & * & 0.3293 \\
\hline Brix & 1.12 & 0.8486 & 0.48 & \multicolumn{2}{|c|}{0.2759} & 14 & 0.0567 & 1.58 & 0.6747 & * & \multicolumn{2}{|c|}{0.3557} & 2.166 & $* *$ \\
\hline Nutrient & $\begin{array}{c}\text { Color } \\
\text { Uniformity }\end{array}$ & $\begin{array}{c}\operatorname{Pr}> \\
\text { Chi Sq }\end{array}$ & $\begin{array}{l}\text { Overall } \\
\text { flavor }\end{array}$ & $\begin{array}{c}\operatorname{Pr}> \\
\text { Chi Sq }\end{array}$ & $\begin{array}{c}\text { Fruit } \\
\text { firmness }\end{array}$ & $\begin{array}{c}\operatorname{Pr}> \\
\text { Chi Sq }\end{array}$ & $\begin{array}{c}\text { Overall } \\
\text { Mouthfeel }\end{array}$ & $\begin{array}{c}\operatorname{Pr}> \\
\text { Chi Sq }\end{array}$ & $\begin{array}{c}\text { Fruit } \\
\text { Juiciness }\end{array}$ & $\begin{array}{c}\operatorname{Pr}> \\
\text { Chi Sq }\end{array}$ & $\begin{array}{l}\text { Overall } \\
\text { Taste }\end{array}$ & $\begin{array}{c}\operatorname{Pr}> \\
\text { Chi Sq }\end{array}$ & Texture & $\begin{array}{c}\operatorname{Pr}> \\
\text { Chisq }\end{array}$ \\
\hline $\begin{array}{l}\text { Ascorbic } \\
\text { Acid }\end{array}$ & 0.23 & 0.1088 & 0.69 & $* *$ & 1.04 & 0.6835 & 0.91 & 0.3975 & 0.53 & 0.0678 & 0.82 & 0.0852 & 0.84 & 0.1933 \\
\hline Tocopherol & * & 0.244 & * & 0.105 & 0.50 & 0.5736 & 0.08 & 0.1178 & * & 0.065 & 0.01 & 0.0263 & 0.12 & 0.2086 \\
\hline $\begin{array}{c}\text { Total } \\
\text { Phenolics }\end{array}$ & 1.00 & 0.9202 & 1.30 & 0.2075 & 1.01 & 0.6938 & 1.03 & 0.2886 & 1.04 & 0.1425 & 1.03 & 0.2587 & 1.03 & 0.2863 \\
\hline Moisture & 17.96 & 0.097 & 1.43 & $* *$ & 1.23 & 0.1577 & 1.32 & 0.0925 & 1.38 & 0.0562 & 1.86 & 0.008 & 1.20 & 0.258 \\
\hline Glucose & * & 0.1314 & * & 0.1731 & * & 0.0554 & * & 0.0431 & * & 0.1862 & 0.01 & 0.417 & * & 0.1582 \\
\hline Brix & * & 0.3928 & 1.54 & 0.522 & 2.30 & 0.2204 & 1.46 & 0.5919 & 0.37 & 0.163 & 0.92 & 0.9065 & 0.97 & 0.9605 \\
\hline
\end{tabular}

"Extreme value, model not well fit; ${ }^{* *}$ Indicates $\mathrm{p}>0.05$, globe model is not significant.

\section{Discussion}

Hydroponic growing methods are growing in popularity and seem to have a positive overtone because of the numerous environmental benefits of soilless methods compared to soil grown crops [1]. Although there are numerous benefits to the hydroponic system, it does not automatically guarantee a high quality product. As this technology is advancing, it is important to consider the sensory attributes of the hydroponic product since taste is one of the main drivers of consumption [18]. Our study indicated $87 \%$ of the participants could correctly identify unspecified differences between hydroponically and soil grown strawberries. Since our participants were untrained, it can be assumed that consumers, in general, may also be able to identify unspecified differences. Unspecified preference tests indicated that $70 \%$ preferred the hydroponically grown berry compared to the soil grown berry; however, the results were not significant $(p=0.06)$. Additionally, the majority of the sensory analysis categories indicated higher ratings for hydroponically grown strawberries, but significance was reached only with aroma and aroma intensity. Our study sample was limited to students enrolled in classes at UNR and the majority of study participants were between the ages of 18 - 29 years old. Larger studies from the general population should be conducted with a random sample to diversify the demographics and make the results more generalizable.

The correlational analysis showed several significant associations with the amount of sweetness in soil and hydroponic strawberries. Additionally, significant associations were observed with overall flavor and overall taste with soil grown strawberries and overall taste with hydroponic strawberries. Although there is a high level 
of variability in consumer acceptance and preference with fruit, it is essential to identify key sensory properties that drive preference. In the correlational analysis, it appears sweetness, flavor and taste correlated with several nutrients and sensory ratings. The results indicate the nutritional composition of the berry may influence sensory scores. With hydroponic food production, it is possible to change parameters in the solution to encourage the plant development of sugars or bioactive compounds. Identification of nutrition factors driving sensory ratings may aid in the development in hydroponic produce production. Additionally, researching these factors in future studies will be important in determining the success of the hydroponic product.

Although this study shows significant findings for nutrient and descriptive sensory data, it is limited with the use of untrained panelists. Untrained panelists are generally considered to be less accurate than trained panelists when using descriptive testing, since they are not oriented to the sensory attributes of interest and there will likely be high variability in the data [19]. However, recent research has suggested this variation might not be as large as previously thought and indicated untrained panelists may be appropriate for descriptive testing [20]. Additional research should be conducted with trained panelists to confirm the findings of this study.

\section{Conclusion}

Findings from this study suggest that consumers do not have significant preference between hydroponic and soil grown strawberries. However, due to the small sample size, further research should be conducted with larger panels of different demographics to verify findings. With the environmental benefits of hydroponic produce production combined with the favorable descriptive sensory analysis ratings, it may be desirable to the consumer and beneficial to the environment to grow strawberry cultivars in non-arable regions to provide fresh fruit. However, much more research should be done to determine the optimum feasibility as well as methods to improve sensory evaluation scores.

\section{Acknowledgements}

We greatly appreciate the Nevada Agricultural Experiment Station, University of Nevada, Reno for the support of this study. HATCH \#0745. The research was done and reported to partially fulfill dissertation requirement of C. Treftz.

\section{References}

[1] Resh, H.M. and Howard, M. (2012) Hydroponic Food Production: A Definitive Guidebook for the Advanced Home Gardener and the Commercial Hydroponic Grower. St. Bárbara, EUA. http://dx.doi.org/10.1201/b12500

[2] Gruda, N. (2009) Do Soilless Culture Systems Have an Influence on Product Quality of Vegetables ? 147, $141-147$.

[3] Treftz, C. and Omaye, S.T. (2015) Nutrient Analysis of Soil and Soilless Strawberries and Raspberries Grown in a Greenhouse. Food and Nutrition Sciences, 6, 805-815. http://dx.doi.org/10.4236/fns.2015.69084

[4] Buchanan, D.N. and Omaye, S.T. (2013) Comparative Study of Ascorbic Acid and Tocopherol Concentrations in Hydroponic- and Soil-Grown Lettuces. Food and Nutrition Sciences, 4, 1047-1053. http://dx.doi.org/10.4236/fns.2013.410136

[5] Mattheis, J.P. and Fellman, J.K. (1999) Preharvest Factors Influencing Flavor of Fresh Fruit and Vegetables. Postharvest Biology and Technology, 15, 227-232. http://dx.doi.org/10.1016/S0925-5214(98)00087-8

[6] Ng, M., Chaya, C. and Hort, J. (2013) The Influence of Sensory and Packaging Cues on Both Liking and Emotional, Abstract and Functional Conceptualisations. Food Quality and Preference, 29, 146-156. http://dx.doi.org/10.1016/j.foodqual.2013.03.006

[7] Garruti, S. and Virginia, H. (2010) Sensory Evaluation in Fruit Product Development.

[8] Rao, A.R. (2005) The Quality of Price as a Quality Cue. Journal of Marketing Research, 42, 401-405. http://dx.doi.org/10.1509/jmkr.2005.42.4.401

[9] Bronnenberg, B.J., Dhar, S.K. and Dubé, J.-P. (2007) Consumer Packaged Goods in the United States: National Brands, Local Branding. Journal of Marketing Research, 44, 4-13. http://dx.doi.org/10.1509/jmkr.44.1.4

[10] Macht, M. (2008) How Emotions Affect Eating: A Five-Way Model. Appetite, 50, 1-11. http://dx.doi.org/10.1016/j.appet.2007.07.002

[11] Steinmetz, K.A. and Potter, J.D. (1996) Vegetables, Fruit, and Cancer Prevention: A Review. Journal of the American Dietetic Association, 96, 1027-1039. http://dx.doi.org/10.1016/S0002-8223(96)00273-8 
[12] Giampieri, F., Tulipani, S., Alvarez-Suarez, J.M., Quiles, J.L., Mezzetti, B. and Battino, M. (2012) The Strawberry: Composition, Nutritional Quality, and Impact on Human Health. Nutrition, 28, 9-19. http://dx.doi.org/10.1016/j.nut.2011.08.009

[13] O’Mahony, M. (2013) The Tetrad Test: Looking Back, Looking Forward. Journal of Sensory Studies, 28, 259-263. http://dx.doi.org/10.1111/joss.12045

[14] Beckley, J.H., Moskowitz, H.R. and Resurreccion, A.V.A. (2006) Sensory and Consumer Research in Food Product Design and Development. Wiley-Blackwell, Oxford. http://dx.doi.org/10.1002/9780470277706

[15] Meilgaard, M.C., Carr, B.T. and Civille, C.G. (2006) Sensory Evaluation Techniques. 4th Edition, CRC Press, Boca Raton.

[16] O’Mahony, M. and Rousseau, B. (2003) Discrimination Testing: A Few Ideas, Old and New. Food Quality and Preference, 14, 157-164. http://dx.doi.org/10.1016/S0950-3293(02)00109-X

[17] Ennis, J.M. and Jesionka, V. (2011) The Power of Sensory Discrimination Methods Revisited. Journal of Sensory Studies, 26, 371-382. http://dx.doi.org/10.1111/j.1745-459X.2011.00353.x

[18] Ho, L.C. (2004) The Contribution of Plant Physiology in Glasshouse Tomato Soilless Culture. Acta Horticulturae, 19-25. http://dx.doi.org/10.17660/ActaHortic.2004.648.2

[19] Stone, H. and Sidel, J.L. (2004) Sensory Evaluation Practices. Academic Press, London.

[20] Worch, T., Lê, S. and Punter, P. (2010) How Reliable Are the Consumers? Comparison of Sensory Profiles from Consumers and Experts. Food Quality and Preference, 21, 309-318. http://dx.doi.org/10.1016/j.foodqual.2009.06.001 